\title{
Orientaçóes emergenciais para profissionais que assistem pacientes com Esclerose Lateral Amiotrófica
}

\author{
Emergency guidelines for professionals who treat patients with Amyotrophic Lateral Sclerosis \\ Leticia Alves Fonseca ${ }^{1}$, Sissy Veloso Fontes', Isabela Pessa Anequini', \\ Francis Meire Fávero 4 , Acary Souza Bulle Oliveira
}

\begin{abstract}
RESUMO
Objetivo. Este artigo teve como objetivo realizar uma revisão da literatura sobre orientaçôes emergenciais em Esclerose Lateral Amiotrófica (ELA) para profissionais da área da saúde. Método. Realizou-se uma revisão da literatura dos últimos dez anos. Foram pesquisados nas bases de dados LILACS, SCIELO, MEDLINE, PEDRO e PUBMED artigos de revisão sistemática, guidelines, manuais de associaçôes, documentos eletrônicos e outros estudos. Resultados. A grande maioria dos manuais encontrados aborda as alteraçóes que podem ser observadas no paciente com ELA como: alteraçáo do sono, úlceras e lesões de pele, sialorreia, disartria, disfagia, mas não enfatiza os procedimentos a serem tomados pelos profissionais da área da saúde quando seus pacientes apresentam tais sintomas, excluindo itens essenciais nos cuidados emergenciais aos pacientes com ELA como: cuidados com a oxigenação, intubação orotraqueal, ventilação mecânica invasiva, traqueostomia, desmame, gastrostomia endoscópica percutânea, entre outros. Conclusáo. Através da pesquisa em questáo verificou-se que a literatura é escassa e sugere-se a elaboração de um manual amplo de orientaçóes emergenciais que aborde os principais cuidados que os profissionais da área da saúde devem realizar em uma situação de emergência em âmbito hospitalar ou domiciliar.
\end{abstract}

Unitermos. Esclerose Amiotrófica Lateral, Aconselhamento Diretivo, Serviços Médicos de Emergência.

Citação. Fonseca LA, Fontes SV, Anequini IP, Fávero FM, Oliveira ASB. Orientaçóes emergenciais para profissionais que assistem pacientes com Esclerose Lateral Amiotrófica.

\begin{abstract}
Objective. This article attempts a literature review of guidelines on emergency Amyotrophic lateral sclerosis (ALS) for health professionals. Method. We conducted a literature review and the last ten years. We searched in the databases LILACS, SciELO, MEDLINE, PEDRO and PUBMED articles of the systematic review, guidelines, manuals associations, electronic documents and other studies. Results. Most of the manuals found address changes that can be observed in patients with ALS as sleep disorders, ulcers and skin lesions, drooling, dysarthria, dysphagia, but does not emphasize the procedures to be taken by health professionals when their patients have such symptoms, other essential items in the emergency care of patients with ALS as: caring for the oxygenation of these patients, invasive mechanical ventilation, tracheostomy, weaning, among others. Conclusion. Through the research in question was found that the literature is scarce and it is suggested the elaboration of a comprehensive emergency guide book that addresses the primary care that health professionals should perform in an emergency situation in the hospital or at home.
\end{abstract}

Keywords. Amyotrophic Lateral Sclerosis, Directive Counseling, Emergency Medical Services.

Citation. Fonseca LA, Fontes SV, Anequini IP, Fávero FM, Oliveira ASB. Emergency guidelines for professionals who treat patients with Amyotrophic Lateral Sclerosis.
Trabalho realizado no Setor de Investigaçáo de Doenças Neuromusculares da Disciplina de Neurologia Clínica da Universidade Federal de Sáo PauloUnifesp, São Paulo-SP, Brasil.

1. Fisioterapeuta, Mestranda do Programa de Ciências de reabilitação da Universidade nove de Julho-UNINOVE, São Paulo-SP, Brasil.

2. Fisioterapeuta, Doutora em Ciências pela UNIFESP, Sáo Paulo-SP, Brasil.

3. Fisioterapeuta, Especialista em Fisioterapia nas Doenças neuromuscularesUNIFESP, São Paulo-SP, Brasil.

4. Fisioterapeuta, Mestre, Coordenadora do Curso de Especialização em Intervenção Fisioterapêutica nas Doenças Neuromusculares da UNIFESP, São Paulo-SP, Brasil.

5. Neurologista, Doutor, Professor Afiliado da Disciplina de Neurologia da UNIFESP, São Paulo-SP, Brasil.
Endereço para correspondência: Leticia Alves Fonseca Rua Domingos de Moraes, $n^{\circ}$ 2818/ 61, Vila Mariana CEP 04036-100, São Paulo-SP, Brasil. E-mail: Leticiaf_alves@hotmail.com 


\section{INTRODUÇÃO}

A Esclerose Lateral Amiotrófica (ELA) foi primeiramente descrita por Charcot em 1869 e tornou-se bastante conhecida como doença de Lou Gehrig devido ao jogador americano de baseball que faleceu com a doença em $1941^{1}$.

É uma doença neurodegenerativa progressiva do SNC, associada à degeneração e perda de neurônios motores superiores e inferiores ${ }^{1}$.

A incidência de ELA nos Estados Unidos é de cerca de dois casos a cada 100.000 pessoas/ano. Estima-se que, no Brasil, a incidência seja semelhante, mas a metade dessas pessoas não sabe que é portadora da doença. Dentre aquelas que recebem o diagnóstico, muitas não obtêm tratamento e orientaçóes adequadas ${ }^{2}$. O diagnóstico é clínico, complementado por testes laboratoriais, eletroneuromiografia e exames de neuroimagem ${ }^{3}$.

Os brancos são mais afetados que os negros ${ }^{1}$. Em um estudo realizado pela Abrela e pelo laboratório Aventis em 1998, foram catalogados 540 pacientes com ELA, sendo 58,5\% homens; 5,9\% dos pacientes tinham história familiar. A idade média de aparecimento dos primeiros sintomas foi 52 anos. Estima-se, em nosso meio, uma incidência de 1,5 casos/100.000 pessoas, ou seja, 2.500 pacientes por $\mathrm{ano}^{2}$.

Há descrição na literatura de três tipos de ELA, esporádica ( $90 \%$ dos casos), familiar $(9 \%)$, e um tipo mais raro, o da ilha de Guam, que representa $1 \%$ dos casos. Vinte por cento das famílias com ELA de herança dominante estão associados a mutaçôes na região q21 do cromossomo 21, que contém o gene da cu/Zn superóxido desmutase 1 (SOD1) que se localiza no cromossomo 21 (21q22.1) ${ }^{4}$.

A etiologia ainda é indefinida, mas acredita-se ser multifatorial e inclui fatores genéticos e ambientais. Existem várias hipóteses etiológicas para ELA, as mais citadas são: mutaçâo da enzima catalizadora superoxido-dismutase (SOD-1); excitotoxicidade com aumento do glutamato; fatores virais e stress oxidativo; fatores ambientais e exposição a determinadas substâncias químicas que agiriam no receptor de glutamato, como chumbo, alumínio e mercúrio 5 .

O quadro clínico da ELA caracteriza-se pela presença de sinais de acometimento do neurônio superior e inferior, associando o quadro de paresia, atrofia e fas- ciculações, hiperreflexia, espasticidade, cãibras e sinal de Babinski. O comprometimento bulbar caracteriza-se por disfonia, disfagia, com paresia, atrofia e fasciculaçóes da musculatura de língua, associados a sintomas como labilidade emocional e depressão, provenientes do acometimento do neurônio motor superior; além do envolvimento da musculatura respiratória cursando com distúrbio ventilatório restritivo grave ${ }^{6}$. Na maioria dos casos, as funçôes cognitivas do paciente permanecem completamente intactas, a despeito do efeito devastador que ocorre no corpo. Pode acontecer dos indivíduos acometidos apresentarem demência fronto-temporal (DFT), que afeta os lobos frontal e temporal do cérebro, visto que tanto a ELA quanto a DFT são afetadas pela mesma proteína TDP-43, sendo a DFT causa de demência pré-senil mais comum em pessoas com menos de 65 anos depois da doença de Alzheimer ${ }^{7}$.

Os músculos ventilatórios são comprometidos e os indivíduos apresentam restriçóes pulmonares, caracterizadas por redução da capacidade vital (CV) e do volume corrente (VC), com consequente insuficiência respiratória crônica ${ }^{8}$ A utilização da ventilação não invasiva (VNI) nos pacientes com ELA tem sido empregada nos últimos anos com o objetivo de corrigir a insuficiência respiratória e, por conseguinte, melhorar a qualidade de vida e prolongar a sobrevivência destes indivíduos?

A intervenção de uma equipe multidisciplinar tem como principal objetivo preservar a qualidade de vida e a funcionalidade. Apesar de a sobrevida média ser de 2 a 5 anos, há grupos de pacientes com mais de uma década, sendo portanto prevista uma longa e criteriosa assistência ${ }^{10}$.

O medicamento utilizado pela maioria destes pacientes é o Riluzole, um antagonista da liberação do glutamato na fenda sináptica e deve começar a ser utilizado na fase inicial da doença. Os efeitos colaterais são já bem conhecidos (efeitos gastrointestinais, astenia, parestesias, tonteira, elevação das enzimas hepáticas) e não existe um consenso de quando interromper a terapia, já que ela não se mostra útil nas fases tardias, aumentando a sobrevida apenas em meses ${ }^{11}$.

A fisioterapia é necessária durante todo o decorrer da doença, com modificações e adaptaçóes essenciais para cada estágio da enfermidade em ordem de retardar a evolução e minimizar os sintomas ${ }^{12,13}$. 
É importante também a orientação dos pacientes, oferecendo programas comunitários como palestras ou cartilhas impressas com explicativos de tratamentos e cuidados, para que estes pacientes possam entender sua patologia em uma linguagem acessível e seguir o tratamento adequado, com o objetivo de melhorar sua qualidade de vida ${ }^{14}$.

A sociedade canadense de ELA elaborou um manual para as pessoas que convivem com a afecção no qual explicam o que é a doença, os sintomas, como se deve lidar com a mesma, como procurar ajuda, entre outros $\operatorname{aspectos}^{15}$. Foi elaborado um manual para pacientes e cuidadores no qual se aborda apenas os cuidados respiratórios destes pacientes ${ }^{16}$.

A morte por ELA geralmente é resultante da deficiência respiratória, a apresentação clínica habitual caracteriza-se por uma diminuição progressiva da força muscular com desenvolvimento de insuficiência respiratória global e infecçóes respiratórias de repetição com alto índice de internação destes pacientes, sobretudo nos cuidados da unidade de terapia intensiva em uma fase mais tardia da doença com 50\% dos pacientes sobrevivendo apenas três a quatro anos depois do início dos sintomas, com uma mortalidade maior nos pacientes com o comprometimento bulbar ${ }^{17}$. Este artigo teve como objetivo realizar uma revisão da literatura sobre as orientaçôes emergenciais para pacientes com ELA para profissionais da área da saúde.

\section{MÉTODO}

Realizou-se uma revisão da literatura (revisão bibliográfica analítica). Para o estudo em questão foram realizadas buscas de artigos científicos na base de dados do LILACS (Literatura Latino-Americana e do Caribe em Ciências da Saúde), SCIELO (The Scientific Electronic Library Online), MEDLINE (Medlars Online), PEDRO (physiotherapy evidence database), sendo considerados somente artigos científicos publicados nos últimos dez anos.

Foram pesquisados estudos do tipo revisão sistemática, guidelines, manuais de associaçóes, documentos eletrônicos e outros estudos. Durante a pesquisa foram utilizados descritores da língua portuguesa: doenças dos neurônios motores, esclerose lateral amiotrófica, protocolo, guia, manuais, orientaçóes, emergência, UTI, prontosocorro; descritores da língua inglesa: amyotrophic lateral sclerosis, protocol, guidelines, motor neuron disease; e descritores da língua espanhola: enfermedad de La neurona motora, esclerosis lateral amiotrófica.

\section{RESULTADOS}

Encontrou-se na literatura pesquisada três guidelines, quatro artigos científicos, dois manuais, uma monografia de conclusão de mestrado, um trabalho de conclusão de curso e um documento eletrônico nos quais, foram abordados cuidados emergências no paciente com ELA (Tabela 1).

\section{DISCUSSÃO}

Não foi encontrado na literatura um trabalho que abordasse todos os principais cuidados emergencias em ELA para profissionais da área da saúde. A associação portuguesa de doentes neuromusculares disponibiliza em seu site um manual sobre ventilação mecânica onde se explica o que é um ventilador e como é utilizado, mas somente na residência do paciente, não relatando, portanto, o uso da ventilação mecânica nos postos de saúde e hospitais e nem parâmetros utilizados no doente neuromuscular.

A sociedade canadense de ELA criou um guideline para pessoas que convivem com ELA, o mesmo contém explicações sobre a doença, onde encontrar ajuda, sinais e sintomas, mobilidade e independência, entre outros itens voltados para o paciente e cuidadores não retratando, portanto, as condutas a serem tomadas pelos profissionais da área da saúde em casos de emergência ${ }^{15}$.

Nos episódios de falência respiratória é comum o paciente chegar aos departamentos de emergências onde profissionais da área de saúde, desconhecendo o conceito de falência ventilatória tendem a tratar os sintomas com a administração de oxigênio. Isto leva a uma exacerbação da hipoventilação e falência súbita com consequente necessidade de intubação (raramente necessária para estes pacientes) ou óbito. Nestes casos deve-se lembrar que o importante é ventilar e não oxigenar o paciente.

Não foram encontrados também nos trabalhos estudados itens abordando os cuidados com a oxigenação do paciente, assim como, parâmetros utilizados na ventilação não invasiva, como proceder com a VNI na emergência, o que pode levar um paciente com ELA ao pronto-socorro, entre outros aspectos relevantes. 
Distribuição de informaçōes sobre ELA em condiçōes emergenciais encontrados na literatura

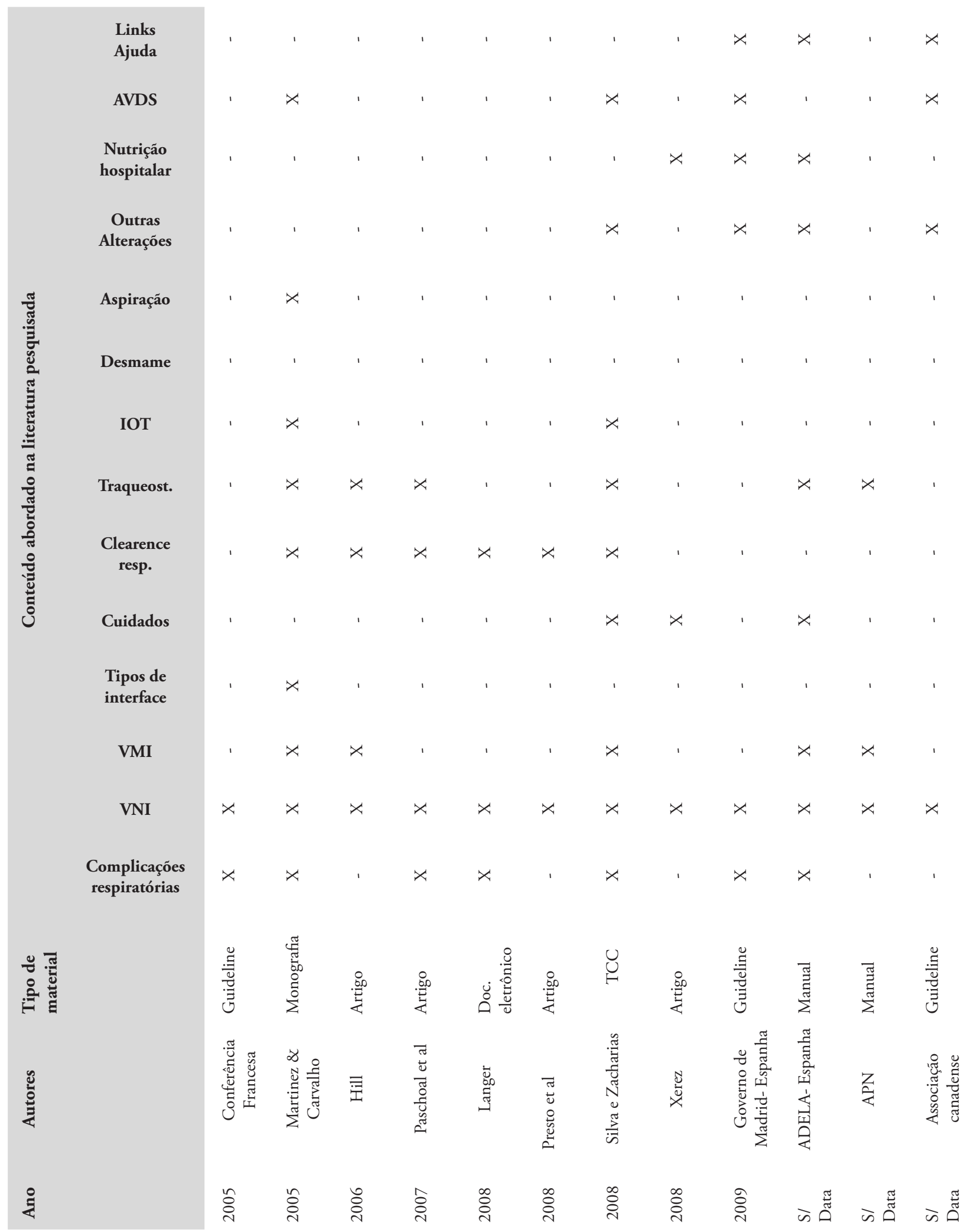


A conferência francesa criou um guideline para cuidados com os pacientes com ELA onde eles relatam a assistência ventilatória (VNI) em domicílio e falência respiratória, não explicando, portanto quais parâmetros utilizados na VNI nestes pacientes e nem quando se torna necessário o uso da ventilação diurna ${ }^{18}$.

O guideline feito pelo governo de Madrid na Espanha possui bastante informaçóes sobre o paciente com ELA e explicaçôes sobre VNI, falência respiratória, alimentação, comunicação, traqueostomia, porém não relata sobre a ventilação invasiva, os tipos de interface, auxílio a tosse, o desmame, entre outros cuidados a serem tomados nas condiçóes emergenciais ${ }^{19}$.

Ressalta-se a importância do auxílio manual e mecânico a tosse através do air-stacking, prensa abdominal ou cought assist (auxiliar da tosse) feitos por profissionais da área da saúde quando o paciente não atinge o fluxo mínimo de tosse: 160 1/min ou 2,7 1/seg o que não foi possível ser encontrado detalhadamente nos manuais pesquisados.

A grande maioria dos trabalhos encontrados aborda algumas alteraçóes que podem ser observadas no paciente com ELA como: alteração do sono, úlceras e lesôes de pele, sialorreia, disartria e disfagia, mas não enfatiza os sinais indicadores de tais situaçóes e os procedimentos a serem tomados pelos profissionais da área da saúde quando seus pacientes com ELA apresentam tais sintomas.

Em boa parte dos trabalhos pesquisados é citado o uso da gastrostomia endoscópica percutânea (GEP), mas não é comumente enfatizado quando se torna necessário o uso e o que pode substituir a GEP no caso dos pacientes que não aceitam esta via de alimentação.

Sugere-se também um manual que enfatize quando está indicado a utilização da intubação orotraqueal (IOT) em casos de emergência, assim como, a atuação da fisioterapia em pacientes neste procedimento, sendo descrito que muitos profissionais frente a uma insuficiência respiratória tendem a utilizar a IOT, o que pode levar a produção excessiva de secreçôes, dificuldade de eliminação, aspiraçóes frequentes, e em casos mais graves ocorrendo intubação seletiva causando pneumotórax. Muitas vezes não há necessidade da IOT e sim da instalação da VNI quando este não estiver utilizando ou quando já faz uso, adequar esta ventilação, aumentando o clearence de secreção $^{16,20}$.
É de extrema importância que seja enfatizado o desmame da VMI, os sinais indicadores que norteiam os profissionais da área da saúde e os modos de desmame mais utilizados em pacientes com doença neuromuscular o que não foi observado em nenhum dos trabalhos estudados.

Os trabalhos disponíveis também não abordam as orientaçóes de como a família deve proceder nos casos de óbito em domicílio.

\section{CONCLUSÃO}

Através desta pesquisa verificou-se escassez na literatura de guidelines sobre a atuação nas situaçóes emergências em âmbito ambulatorial ou hospital dos pacientes com ELA.

Sugere-se, portanto a elaboração de um manual emergencial de cuidados em ELA para nortear as condutas dos profissionais da área de saúde.

\section{REFERÊNCIAS}

1.Pozza AM, Delamura MK, Ramirez C, Valério NI, Marino LHC, Lamari NM. Conduta Fisioterapêutica na esclerose lateral amiotrófica. Sao Paulo Med J 2006;124:350-4.

http://dx.doi.org/10.1590/S1516-31802006000600011

2.Piemonte M E. Manual de Exercícios Domiciliares para Pacientes com Esclerose Lateral Amiotrófica. Vol. 2. São Paulo: Manole, 2001, 40p.

3.Le Forestier N. Diagnostics différentiels de la sclerose latérale amyotrophique. [The differential diagnosis of amyotrophic lateral sclerosis]. Rev Med Liege 2004;59(Suppl 1):25-31.

4.Wicklund MP. Amyotrophic lateral sclerosis: possible role of environmental influences. Neurol Clin 2005; 23:461-84.

http://dx.doi.org/10.1016/j.ncl.2004.12.016

5.Hosler BA, Brow RH. Cooperlzinc superoxide dismutase mutations and free radical damage in amyotrophic lateral sclerosis. In: Serratrici GT, Munsat TL. Advances in neurol. Philadelphia: Raven Puleiches 1995, p.41-6.

6.Boille S, Velde CV, Cleveland DW. ALS: a disease of motor neurons and their nonneuronal neighbors. Neuron 2006;52:39-59.

http://dx.doi.org/10.1016/j.neuron.2006.09.018

7.Burst JCM. A prática da neurociência: das sinapses aos sintomas. Rio de Janeiro: Reichmann \& Afonso, 2000, p.75-93.

8. Kelly BJ, Lucce JM. The diagnosis and management of the neuromuscular diseases causing respiratory failure. Chest 1991;99:1485-94.

http://dx.doi.org/10.1378/chest.99.6.1485

9.Lo Coco D, Marchese S, Pesco MC, La Bella V, Piccoli F, Lo Coco A. Noninvasive positive-pressure ventilation in ALS- Predictors of tolerance and survival. Neurology 2006;67:761-5.

http://dx.doi.org/10.1212/01.wnl.0000227785.73714.64

10.Orsini M, Freitas MRG, Mello MP, Antonioli RS, Reis JPB, Nascimento OJM, et al. Reabilitação física na esclerose lateral amiotrófica. Rev Neurocienc 
2009;17:30-6.

11.Bryson HM, Fulton B, Benfield P, Riluzole. A review of its pharmacodynamic and pharmacokinetic properties and therapeutic potential in amyotrophic lateral sclerosis. Drugs 1996;52:549-63.

http://dx.doi.org/10.2165/00003495-199652040-00010

12.Chieia MAT, Pereira RDB, Oliveira ASB, Quadros AAJ, Fernandes E. Livreto Informativo do XVI Simpósio Internacional de ELA/DNM, São Paulo: Gráfica RJR, 2007, 20p.

13.Dalbello HV, Kloos AD, Mitsumoto H. Physical therapy for a patient through six stages of amytrophic lateral sclerosis. Phys Ther 1998;78:1312-24. 14.Mitsumoto $\mathrm{H}$, Bene MD. Improving the quality of life for people with ALS: The challenge ahead. Amyotroph Lateral Scler Other Motor Neuron Disord 2000;2:329-36.

http://dx.doi.org/10.1080/146608200300079464

15.Manual for People Living with ALS. Toronto- Ontario, The ALS society of Canada. [acessado em: 02/2009; atualizado em: 02/2009]. Disponível em: http://www.als.ca/als_manuals.aspx
16.Martinez AM, Carvalho EV. Proposta do conteúdo a ser abordado em um manual sobre os cuidados respiratórios para pacientes com esclerose lateral amiotrófica e seus cuidadores [monografia]. São Paulo, SP: UNIFESP, 2005, p.43.

17.Perrin C, Unterborn JN, Ambrosio CD, Hill NS. Pulmonary complications of chronic neuromuscular diseases and their management. Muscle Nerve 2004;29:5-27.

http://dx.doi.org/10.1002/mus.10487

18.Société Française de Neurologie. Care of patients with amyotrophic lateral sclerosis. Nice-France. [acessado em: 02/2009; atualizado em: 02/2009]. Disponível em: www.has-sante.fr

19.Guía para la Atención de la Esclerosis Lateral Amiotrófica (ELA). MadridEspanha. Centro de Publicação Paseo del Prado. [acessado em: 01/2009; atualizada em 09/2009]. Disponível em: http://www.msc.es/profesionales/prestacionesSanitarias/publicaciones/ELA.htm

20.Silva CS, Zacharias S. Atuação da Fisioterapia em Pacientes com Esclerose Lateral Amiotrófica nos serviços de urgência e emergência [Trabalho de Conclusão de Curso]. São Bernardo dos Campos; UNIMEP, 2008, p.56. 